\title{
NOTE ON HYPOELLIPTICITY OF A FIRST ORDER LINEAR PARTIAL DIFFERENTIAL OPERATOR
}

\author{
YOSHIO KATO
}

§1. Introduction. Let $\Omega$ be a domain in the $(n+1)$-dimensional euclidian space $R^{n+1}$. A linear partial differential operator $P$ with coefficients in $C^{\infty}(\Omega)^{1)}$ (resp. in $C^{\omega}(\Omega)^{1)}$ ) will be termed hypoelliptic (resp. analytichypoelliptic) in $\Omega$ if a distribution $u$ on $\Omega$ (i.e. $u \in \mathscr{D}^{\prime}(\Omega)$ ) is an infinitely differentiable function (resp. an analytic function) in every open set of $\Omega$ where $P u$ is an infinitely differentiable function (resp. an analytic function).

In the present paper, we consider a linear partial differential operator

$$
L=\sum_{j=1}^{n+1} a^{j}(y) \frac{\partial}{\partial y^{j}}+a(y),
$$

where the coefficients are complex-valued infinitely differentiable functions (or complex-valued analytic functions) in a domain $\Omega$ of $R^{n+1}$.

Now the main result is :

THEOREm. Suppose that $n \geqslant 2 . \quad$ A linear partial differential operator of the form (1) with coefficients in $C^{\infty}(\Omega)$ (resp. in $C^{\omega}(\Omega)$ ) is hypoelliptic (resp. analytichypoelliptic) in $\Omega$ if and only if all the functions $a^{j}(y) \quad(j=1, \cdots, n+1)$ identically vanish in $\Omega$ and the function a(y) vanishes at no point of $\Omega$.

For $n=1$, the hypoellipticity and the analytic-hypoellipticity of the operator of the form (1) with coefficients in $C^{\omega}$ are characterized by H. Suzuki [4] under the condition $\left|a^{1}(y)\right|+\left|a^{2}(y)\right| \neq 0$ for every $y \in \Omega$ and $a(y)=0$ in $\Omega$.

In the next section, we shall first show that if $L(n \geqslant 1)$ has coefficients in $C^{\omega}(\Omega)$ and satisfies the condition (3) (see $\S 2$ ), the hypoellipticity of $L$ as well as the analytic-hypoellipticity of $L$ has no respect to the factor $a(y)$ and we shall study relations between the solvability ${ }^{2)}$ and the hypoellipticity of $L$. In the last section, we shall prove the theorem.

Received June 7, 1967.

1) We denote by $C^{\infty}(\Omega)$ the totality of complex-valued infinitely differentiable functions in $\Omega$ and by $C^{\omega}(\Omega)$ the totality of complex-valued analytic functions in $\Omega$.

2) A linear partial differential operator defined on $\Omega$ is called solvable in a subdomain $\Omega_{0}$ of $\Omega$ if the equation $P u=f$ has a solution $u \in \mathscr{D}^{\prime}\left(\Omega_{0}\right)$ for every $f \in C_{0}^{\infty}\left(\Omega_{0}\right)$. 
The author extends his hearty thanks to Prof. T. Kuroda for his kind encouragement.

§ 2. Preliminaries. We denote by $L_{0}$ the principal part of $L$ :

$$
L_{0}=\sum_{j=1}^{n+1} a^{j}(y) \frac{\partial}{\partial y^{j}} \text {. }
$$

In this section, we always assume that

$$
\sum_{j=1}^{n+1}\left|a^{j}(y)\right| \neq 0, \quad \text { for all } y \in \Omega .
$$

We first state the following :

Lemma 1. Suppose that $n \geqslant 1$. An operator $L$ of the form (1) with coefficients in $C^{\omega}(\Omega)$ and satisfying the condition (3) is hypoelliptic (resp. analytichypoelliptic) in $\Omega$, if and only if the operator $L_{0}$ is hypoelliptic (resp. analytichypoelliptic) in $\Omega$.

Proof. Let $y_{0}$ be an arbitrary point of $\Omega$. By the Cauchy-Kovalevsky theorem, we can find a solution $h(y)$, analytic in some neighbourhood $N$ of $y_{0}$, of the equation

$$
L_{0} h=a .
$$

From this, we can deduce

$$
\left\{\begin{array}{l}
L_{0}\left(e^{h} u\right)=e^{h} L u, \\
L\left(e^{-h} u\right)=e^{-h} L_{0} u
\end{array}\right.
$$

for all $u \in \mathscr{D}^{\prime}(N)$. We can immediately conclude Lemma 1 from (4), since the notion of hypoellipticity as well as that of analytic-hypoellipticity has a local property.

$$
\text { Q.E.D. }
$$

We set

$$
\bar{L}_{0}=\sum_{j=1}^{n+1} \overline{a^{j}(y)} \frac{\partial}{\partial y^{j}}
$$

and denote by $C$ the commutator

$$
C=\left[L_{0}, \bar{L}_{0}\right]=L_{0} \bar{L}_{0}-\bar{L}_{0} L_{0} \text {. }
$$

We say $L$ satisfy the condition $\mathrm{H}$ at a point $y_{0}$ of $\Omega$, if $C$ may be 
represented as a linear combination of $L_{0}$ and $\bar{L}_{0}$ at $y=y_{0}$. The Hörmander's necessary condition for $L$ to be solvable in a subdomain $\Omega_{0}$ of $\Omega$ is that $L$ satisfies the condition $\mathrm{H}$ at every point of $\Omega_{0}$ (see Chap. VI of Hörmander [1]).

Lemma 2. Suppose that $n \geqslant 2$. If $L$ with coefficients in $C^{\infty}(\Omega)$ (resp. in $\left.C^{\omega}(\Omega)\right)$ and satisfying the condition (3) fulfils the condition $\mathrm{H}$ at every point of $\Omega$, it then follows that $L_{0}$ is not hypoelliptic (resp. not analytic-hypoelliptic) in $\Omega$ and there exists a subdomain of $\Omega$ where $L$ is solvable.

Proof. The proof was suggested by Nirenberg-Trèves [3]. Let $y_{0}$ be a point fixed arbitrarily in $\Omega$. By a suitable coordinate transformation in some neighbourhood of the point $y_{0}, L_{0}$ may be expressed in the form

$$
L_{0}=g(x, t)\left(\frac{\partial}{\partial t}+i \sum_{j=1}^{n} b^{j}(x, t) \frac{\partial}{\partial x^{j}}\right), \quad g(x, t) \neq 0,
$$

$\left(i=\sqrt{-1}, x=\left(x^{1}, \cdots, x^{n}\right)\right)$ in a neighbourhood $N$ of the origin : $x=0$, $t=0$, so that $L$ is written by the new coordinate as follows:

$$
L=g(x, t)\left(\frac{\partial}{\partial t}+i \sum_{j=1}^{n} b^{j}(x, t) \frac{\partial}{\partial x^{j}}\right)+c(x, t),
$$

where $b^{j}(x, t)(j=1, \cdots, n)$ are real-valued, and the transformation of coordinates and the coefficients of $L$ of the form (5) are both infinitely differentiable (resp. analytic) in $N$, if the coefficients of $L$ of the form (1) is infinitely differentiable (resp. analytic) in $\Omega$ (see [3]).

If $L$ satisfies the condition $\mathrm{H}$ in $N$, it follows that

$$
\sum b_{t}^{j}(x, t) \xi_{j}=0 \quad \text { if } \sum b^{j}(x, t) \xi_{j}=0, \quad(x, t) \in N, \quad \xi \in R^{n},
$$

where $b_{t}^{j}(x, t)=\frac{\partial b^{j}}{\partial t}(x, t)$.

Let $\boldsymbol{b}(x, t)$ be the real vector $\left(b^{1}(x, t), \cdots, b^{n}(x, t)\right)$ and $|\boldsymbol{b}(x, t)|$ be the length of the vector $\boldsymbol{b}$ :

$$
|\boldsymbol{b}(x, t)|=\left(b^{1}(x, t)^{2}+\cdots+b^{n}(x, t)^{2}\right)^{\frac{1}{2}} .
$$

If $|\boldsymbol{b}(x, t)|$ identically vanishes in $N$, any function depending only on the variables $x$ is always a solution of the equation $L_{0} u=0$. Otherwise, we can find a subdomain $N_{1}$ of $N$ in which $\boldsymbol{b}(x, t)$ never vanishes. Thus it follows from (6) that there exists a real-valued function $\beta(x, t)$ in $C^{\infty}\left(N_{1}\right)$ such that 


$$
\boldsymbol{b}_{t}(x, t)=\beta(x, t) \boldsymbol{b}(x, t),
$$

where we have put $\boldsymbol{b}_{t}=\left(b_{t}^{1}, \cdots, b_{t}^{n}\right)$, and from (7) we obtain

$$
\frac{d}{d t}(\boldsymbol{b}(x, t) /|\boldsymbol{b}(x, t)|)=0, \text { in } N_{1} \text {. }
$$

Hence the real vector $\boldsymbol{b}(x, t) /|\boldsymbol{b}(x, t)|$ is independent of the variable $t$. If we put $\boldsymbol{v}(x)=\boldsymbol{b}(x, t) /|\boldsymbol{b}(x, t)|, L_{0}$ is rewritten in the form

$$
L_{0}=g(x, t)\left(\frac{\partial}{\partial t}+|\boldsymbol{b}(x, t)| \sum_{j=1}^{n} v^{j}(x) \frac{\partial}{\partial x^{j}}\right),
$$

where $\boldsymbol{v}(x)=\left(v^{1}(x), \cdots, v^{n}(x)\right)$. Any solution of the equation

$$
\sum_{j=1}^{n} v^{j}(x) \frac{\partial u}{\partial x^{j}}=0
$$

depending only on the variables $x$ is a solution of the equation $L_{0} u=0$. From these fact, we can assert the first half of the lemma and at the same time we can easily see that $L$ has the property (P) (introduced in [3]) in some subdomain $N^{\prime}$ of $N$, that is, there is a unit vector $\boldsymbol{v}=\boldsymbol{v}(\boldsymbol{x})$ depending on the $x$-variable only such that $\boldsymbol{b}$ is given by $\boldsymbol{b}(x, t)=|\boldsymbol{b}(x, t)| \boldsymbol{v}(x)$ in $N^{\prime}$. Thus using Theorem 2.1 of [3], we obtain the later half of the lemma.

Q.E.D.

Lemma 3. Suppose that $n \geqslant 1$. If an operator $L$ of the form (1) with coefficients in $C^{\omega}(\Omega)$ and satisfying the condition (3) does not fulfil the condition $\mathrm{H}$ at some point in $\Omega$, it then follows that $L_{0}$ is not analytic-hypoelliptic in $\Omega$.

Proof. This lemma is easily deduced from Theorem 4.1 of Mizohata [2]. But in our case the proof is simpler. We shall give an outline of the proof.

Suppose that $L$ does not fulfil the condition $\mathrm{H}$ at a point $y_{0} \in \Omega$. Then we can construct a solution $w$ of the equation $L_{0} u=0$ in a neighbourhood $N$ of $y_{0}$ such that $w\left(y_{0}\right)=0$ and the imaginary part of $w$ is positive in $N$, the point $y_{0}$ excepted (see Chap. VI of [1]). If we take a suitable branch, $\sqrt{w(y)}^{3}$ is continuously differentiable in $N$ and satisfies the equation $L_{0} u=0$. But it is not twice-continuously differentiable at $y_{0}$. This gives the proof.

Q.E.D. 
Finally, we state the lemma given by Mr. A. Yoshikawa (see [5]).

Lemma 4. Let $\Omega$ be a domain of $R^{n+1}(n \geqslant 0)$ and $P$ be a general linear partial differential operator with coefficients in $C^{\infty}(\Omega)$. If $P$ is hypoelliptic in $\Omega$, then the formal adjoint ${ }^{t} P$ of $P$ is solvable in a neighbourhood of each point of $\Omega$. Here the differential operator ${ }^{t} P$ is defined by the identity

$$
\int P u \cdot v d y=\int u \cdot{ }^{t} P v d y, \quad u, v \in C_{0}^{\infty}(\Omega) .
$$

Proof. Suppose that $P$ is hypoelliptic in $\Omega$. Let $S$ be the totality of locally square-integrable functions $u$ in $\Omega$ such that $P u$ is in $C^{\infty}(\Omega)$. We note $S=C^{\infty}(\Omega)$ and denote by $G_{P}$ the graph of $P$ on $S$ into $C^{\infty}(\Omega)$ in the product space $L_{\text {loc }}^{2}(\Omega) \times C^{\infty}(\Omega)$, that is, $G_{P}=\{[u, P u] ; u \in S\}$. Then, by the open mapping theorem of Banach, the projection on $G_{P}$ onto $C^{\infty}(\Omega)([u, P u]$ $\rightarrow u)$ is continuous ${ }^{3)}$. Thus let $y_{0}$ be an arbitrary point of $\Omega, N_{0}$ be a neighbourhood of $y_{0}$ whose closure $N_{0}$ is contained in $\Omega$, and $k$ be an arbitrary integer $\geqslant 0$. There then exists a constant $C_{0}$, an integer $s_{0} \geqslant 0$ and compact sets $K_{1}, K_{2}$ of $\Omega$ depending on $k$ and $N_{0}$ such that

$$
|u|_{k, \bar{N}_{0}} \leq C_{0}\left\{\left(\int_{K_{1}}|u|^{2} d y\right)^{\frac{1}{2}}+|P u|_{s_{0}, K_{2}}\right\}, \quad u \in C^{\infty}(\Omega) .
$$

If we choose a neighbourhood $N$ of $y_{0}$ such that $N \subset N_{0}$ and

$$
C_{0} \sqrt{ } \text { Volume of } N \leq \frac{1}{2},
$$

we obtain from (8) that

$$
\|\varphi\|_{k} \leq|P \varphi|_{s_{0}}, \quad \varphi \in C_{0}^{\infty}(N),
$$

where we have put

$$
\|\varphi\|_{k}=\left(\sum_{|\alpha| \leqslant k} \int\left|D^{\alpha} \varphi\right|^{2} d y\right)^{\frac{1}{2}}
$$

3) By $\alpha$ we denote multi-indices $\alpha=\left(\alpha_{1}, \cdots, \alpha_{n+1}\right)$ of non-negative integers. Their sum is denoted by $|\alpha|$. With $D_{j}=-i \partial / \partial y^{j}$, we set

$$
D^{\alpha}=D_{1}^{\alpha_{1}} \cdots D_{n+1}^{\alpha_{n+1}} .
$$

The topology of $C^{\infty}(\Omega)$ is then defined by the semi-norms $|\cdot|_{m, K}$ :

$$
|f|_{m, K}=\sum_{|\alpha| \leqslant m} \sup _{y \in K}\left|D^{\alpha} f(y)\right|,
$$

where $m$ is any non-negative integer and $K$ is any compact set of $\Omega$. Hence $C^{\infty}(\Omega)$ is a Fréchet space by this topology. 
From this we may deduce the inequality

$$
\|\varphi\|_{k} \leq C\|P \varphi\|_{s} \quad, \quad \varphi \in C_{0}^{\infty}(N)
$$

since we have

$$
|\varphi|_{s_{0}} \leq C\|\varphi\|_{s}, \quad \varphi \in C_{0}^{\infty}(N)
$$

with some integer $s>0$ and a constant $C>0$.

From (9) we can immediately see that ${ }^{t} P$ is solvable in a neighbourhood of each point of $\Omega$.

$$
\text { Q.E.D. }
$$

§3. Proof of Theorem. Finally we prove the theorem stated in the introduction. We have only to prove the following :

Proposition. If $n \geq 2$, no operator of the form (1) with coefficients in $C^{\infty}(\Omega) \quad$ (resp. in $C^{\omega}(\Omega)$ ) and satisfying the condition (3) is hypoelliptic (resp. analytichypoelliptic) in $\Omega$.

Before proving the proposition, we must state a lemma which is needed in proving the proposition above.

Lemma 5. Let $M$ be a linear mapping on $C^{\infty}(\Omega)$ onto itself which satisfies the following conditions:

(i) The mapping $M$ is bijective and bicontinuous ${ }^{4)}$.

(ii) A function $u$ belonging to $C^{\infty}(\Omega)$ identically vanishes in a subdomain of $\Omega$ if and only if $M u$ identically vanishes there.

Then $M$ is an operator of multiplication by a non-vanishing function in $C^{\infty}(\Omega)$.

Proof of Lemma 5. It is clear that $M$ and its inverse mapping $M^{-1}$ are both linear partial differential operators with coefficients in $C^{\infty}(\Omega)$ :

$$
\begin{aligned}
& M=P(y, D)=\sum_{|\alpha| \leqslant m_{y}} a_{\alpha}(y) D^{\alpha}, \\
& M^{-1}=Q(y, D)=\sum_{|\alpha| \leqslant n_{y}} b_{\alpha}(y) D^{\alpha},
\end{aligned}
$$

where $m_{y}$ and $n_{y}$ are exact orders of $P(y, D)$ and $Q(y, D)$ at a point $y$ respectively, and they are bounded when $y$ goes over a compact set of $\Omega$.

First of all, we shall show that $m_{y}$ and $n_{y}$ both identically vanish in

4) The topology of $C^{\infty}(\Omega)$ is the same one as the topology stated in footnote 3 ). 
$\Omega$. Assume that $n_{y} \not \equiv 0$ in $\Omega$. There then exists a subdomain $\Omega_{0}$ of $\Omega, \bar{\Omega}_{0} \subset \Omega$, where $n_{y}$ is a positive constant, say $n$. Put $m=\max _{y \in \bar{\Omega}_{0}} m_{y}$. By $P_{m}(y, \xi)$ and $Q_{n}(y, \xi)$, we denote the principal parts of the characteristic polynomials $P(y, \xi)$ and $Q(y, \xi)\left(y \in \Omega_{0}, \xi \in R^{n+1}\right)$ respectively. Clearly we have

$$
P_{m}(y, \xi) Q_{n}(y, \xi)=0
$$

for all $y \in \Omega_{0}$ and all $\xi \in R^{n+1}$. It follows from (10) that $P_{m}(y, \xi)=0$ for all $y \in \Omega_{0}$ and all $\xi \in R^{n+1}$. Hence we have $m=0$. This is a contradiction, since $P_{0}(y, \xi)=(M(1))(y)$ in $\Omega_{0}$. Therefore $n_{y}$ as well as $m_{y}$ identically vanishes in $\Omega$. Thus we can assert that $M$ is equal to an operator of multiplication by a nonvanishing factor. This completes the proof of Lemma 5.

Proof of Proposition. Let $L$ be an operator of the form (1) with coefficients in $C^{\omega}(\Omega)$. Assume that the condition (3) is fulfiled. The lemmas 1,2 and 3 show us that $L$ is not analytic-hypoelliptic in $\Omega$. In the same way, we can deduce from the lemmas 2 and 4 that the principal part $L_{0}$ of an operator $L$ of the form (1) with coefficients in $C^{\infty}(\Omega)$ is not hypoelliptic in any subdomain $\Omega^{\prime}$ of $\Omega$ under the condition (3), since if $L_{0}$ is hypoelliptic in $\Omega^{\prime},{ }^{t} L_{0}$ is solvable in a neighbourhood of each point of $\Omega^{\prime}$ and $L_{0}$ satisfies the condition $\mathrm{H}$ at every point of $\Omega^{\prime}$.

Next, we are going to show that $L$ with coefficients in $C^{\infty}(\Omega)$ is not hypoelliptic in $\Omega$ under the condition (3). Assume that $L$ is hypoelliptic in $\Omega$ and the condition (3) holds. If there exists a solution $v$ of the equation $L v=0$ in a subdomain $\Omega_{1}$ of $\Omega$ such that $v$ does not vanish in $\Omega_{1}$, we can construct a function $h \in C^{\infty}\left(\Omega_{1}\right)$ satisfying

$$
L_{0} h=a \text {. }
$$

In fact we have only to take $h=-\log v$. (Here note that $v$ is in $C^{\infty}\left(\Omega_{1}\right)$ by the assumption on $L$ and that we may, without loss of generality, assume that the range of $v$ is in the upper half-complex plane). By the same method as in the proof of Lemma 1, it follows that $L_{0}$ is hypoelliptic in $\boldsymbol{\Omega}_{1}$. This is a contradiction. Therefore $v$ vanishes in every open set where $L v$ vanishes. On the other hand, by Lemma 4 and the assumption on $L, L$ satisfies the condition $\mathrm{H}$ at each point of $\Omega$. From this and Lemma 
2 , we can conclude that $L$ is solvable in some subdomain $\Omega_{0}$ of $\Omega$. Hence the equation

$$
L u=f
$$

has a solution $u \in C^{\infty}\left(\Omega_{0}\right)$ for every $f \in C_{0}^{\infty}\left(\Omega_{0}\right)$. Thus we can more generally assert that the equation (11) has a unique solution $u \in C^{\infty}\left(\Omega_{0}\right)$ for every $f \in C^{\infty}\left(\Omega_{0}\right)$. Hence $L$ is bijective and continuous mapping on $C^{\infty}\left(\Omega_{0}\right)$ onto itself. By the open mapping theorem of Banach, the inverse mapping of $L$ is also continuous. Therefore we can apply Lemma 5 to $M=L$. That is, $L$ is equal, in $\Omega_{0}$, to an operator of multiplication by a function in $C^{\infty}\left(\Omega_{0}\right)$. Since this contradicts the condition (3), the proof is complete.

Remark. The author was informed that Mr. A. Yoshikawa had proved the following as an application of Lemma $4:$ If $L_{0}$ of the form (2) with coefficients in $C^{\omega}(\Omega)$ satisfying the condition (3) is hypoelliptic in $\Omega$, then $n \leq 1$ (see [5]).

\section{REFERENCES}

[1] L. Hörmander: Linear partial differential operators, Springer-Ver., Berlin (1963).

[2] S. Mizohata: Solutions nulles et solutions non analytiques, J. Math. Kyoto Univ., 1 (1962), 271-302.

[ 3 ] L. Nirenberg and F. Trèves: Solvability of a first order linear partial differential equation, Comm. Pure Appl. Math., 16 (1963), 331-351.

[4] H. Suzuki: Analytic-hypoelliptic differential operators of first order in two independent variables, J. Math. Soc. Japan, 16 (1964), 367-374.

[5] A. Yoshikawa: On the hypoellipticity of differential operators, J. Fac. Sci. Univ. Tokyo, 14 (1967), 81-88.

Department of Mathematics

Aichi University of Education

Okazaki-shi, Aichi-ken ( Japan) 